\title{
Regular group exercise is associated with improved mood but not quality of life following stroke
}

Purpose: People with stroke living in the community have an increased prevalence of depression and lower quality of life than healthy older adults. This cross-sectional observational study investigated whether participation in regular exercise was associated with improved mood and quality of life. Methods: We recruited three groups of community dwelling participants: 13 healthy older adults, 17 adults post-stroke who regularly participated in group exercise at a community fitness facility and 10 adults post-stroke who did not regularly exercise. We measured mood using the Depression, Anxiety, Stress Scale (DASS) and quality of life using the Assessment of Quality of Life (AQoL). Results: Levels of stress and depression were significantly greater in the people with stroke who did not undertake regular exercise $(p=0.004$ and $p=0.004$ respectively), although this group had more recent strokes $(p<0.001)$. Both stroke groups had lower quality of life scores $(p=$ 0.04 ) than the healthy adults. Conclusions: This small, community-based study confirms that people following stroke report poorer quality of life than stroke-free individuals. However, those who exercise regularly have significantly lowerstress and depression compared to stroke survivors who do not. Future research should focus on the precise type and amount of exercise capable of improving mood following stroke. 
1 Michelle N McDonnell ${ }^{1}$, Shylie F Mackintosh ${ }^{1}$, Susan L Hillier ${ }^{1}$ and Janet Bryan².

2 'International Centre for Allied Health Evidence, University of South Australia, Adelaide, South

3 Australia

$4 \quad{ }^{2}$ School of Psychology, Social Work and Social Policy, University of South Australia.

\section{Corresponding author:}

6 Dr Michelle N McDonnell

7 iCAHE

8 University of South Australia

9 GPO Box 2471

10 Adelaide SA 5001

11 Email: michelle.mcdonnell@unisa.edu.au

12 Phone: +61 883021684

13 Fax: +61883022168 
15 Mood disorders have been recognized as a complication following stroke since 1977 (Folstein et

16 al. 1977). Approximately one-third of stroke survivors have mood disorders, with depression and

17 anxiety most frequently measured (Lees et al. 2012). Carers of stroke survivors report

18 disturbances in mood as the most stressful stroke-related problem (․ㅏaley et al. 2009) and despite

19 recommendations published in international guidelines to routinely assess mood post-stroke

20 (Lindsay et al. 2010; National Stroke Foundation 2010), evidence-based interventions for mood

21 disturbances are rarely provided (Foundation 2008).

22 Exercise programs can improve mood through reducing depression and anxiety in healthy adults

23 (Byrne \& Byrne 1993; Howard et al. 2013) and there are pilot data to suggest that a cardiac

24 rehabilitation program for secondary stroke prevention may also decrease depression (Lennon et

25 al. 2008). We investigated the benefits of regular exercise on mood and quality of life in a

26 community setting involving three different groups - those currently exercising who had

27 experienced a stroke, healthy adults without stroke and people with stroke referred to the

28 community centre but not currently exercising.

$29 \quad$ Methods

30 Participants.

31 Participants were recruited from the Centre for Physical Activity in Ageing (CPAA), a unit of a

32 large metropolitan hospital. People living in the surrounding community may be referred to the

33 centre by their medical practitioner or following inpatient or community rehabilitation. Healthy

34 older adults exercising at least once a week (HE) and people with stroke exercising at least once a

35 week (SE) were recruited via flyers posted at the Centre. The third group was recruited via 
36 referrals to the centre for people post-stroke who wished to commence a fitness program but had

37 not commenced exercise classes (STR).

38 As this was a cross-sectional observational study with a sample of convenience, participants were

39 recruited to a group based on their characteristics after which we assessed mood and quality of

40 life. Participants were excluded if they were diagnosed with dementia or if they had insufficient

41 communication skills to complete the questionnaires. There were no age restrictions. This study

42 was approved by relevant ethics committees and participants provided written informed consent

43 in accordance with the Declaration of Helsinki.

44 Assessment Tools.

45 The Montreal Cognitive Assessment (MoCA) was used to screen for mild cognitive impairment

46 (MCI), with a cut-off score of 26 or above indicating normal range (Nasreddine et al. 2005). The

47 Functional Ambulation Category (FAC) was used to classify the ability to ambulate with or

48 without assistance (Mehrholz et al. 2007).

49 Mood disorders were measured using the Depression, Anxiety, Stress Scale (DASS) (Crawford \&

50 Henry 2003) and quality of life using the Assessment of Quality of Life (AQoL) (Hawthorne

51 2000). The DASS measures the three aspects of mood on a Likert scale allowing a maximal score

52 of 42 for each of the three subscales. The DASS exhibits very good reliability (Cronbach's $\alpha=$

530.90 for the anxiety scale, 0.95 for the depression scale and 0.93 for the stress scale score) and

54 internal and convergent validity is high for both depression and anxiety (with the Personal

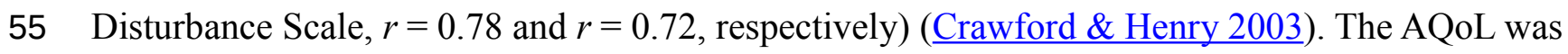

56 used to assess health-related quality of life in five dimensions: independent living, social

57 relationships, physical senses, psychological well-being, and illness. It has been validated for use

58 in people with stroke (Sturm et al. 2002) and scores range from 15-60, with a higher score 
59 indicating poorer quality of life. The AQoL also has sound psychometric properties, with good

60 internal consistency (Cronbach's alpha $\alpha=0.87)$ and test-retest reliability $(r=0.8)$ (Hawthorne

$61 \underline{2000})$.

62

63 The Centre for Physical Activity in Ageing conducts approximately 40 circuit-style classes per

64 week, with 6 dedicated stroke fitness classes and several "slow circuit" classes which are

65 appropriate for people with impaired mobility. People referred to the centre from the community or following rehabilitation are provided with an initial 12 week program which is then reviewed

67 by an exercise physiologist and continued if appropriate. Participants in the exercise groups (HE 68 and SE) attended low to moderate intensity circuit classes, with approximately 20 participants in 69 a class, conducted by a trained fitness instructor. The 60 minute class started with a group warm up (standing or seated if necessary) for 5 minutes and cool down at the end of the class for 5

71 minutes. For the remaining 50 minutes each participant had their own tailored program devised

72 by an exercise physiologist, according to their needs, and supervised by a fitness instructor with a

73 carer present if required to assist with accessing the equipment. A typical class involved

74 strengthening exercises with hydraulic equipment, aerobic exercise with a bike, treadmill or arm

75 ergometer, walking and other weight bearing exercises in the parallel bars, and other higher level

76 activities such as stair climbing or rowing machines. All participants in the SE and HE groups

77 took part in at least one session per week.

78 Statistical analysis

79 Data were inspected for normality and transformed appropriately if required. Group differences

80 were compared using one way analysis of variance (ANOVA; SPSS Version 18) for age, years of

81 education, FAC, MoCA, exercise history, years since stroke, depression, anxiety and stress, and 
82 AQoL, and a Chi-square test for group differences in sex. Levine's test assessed homogeneity of

83 variance for each group. In cases of unequal variances, analysis was repeated using the Welch

84 Robust Test of Equality of Means. Post-hoc analyses following ANOVA were preplanned to

85 assess for differences between groups using Tukey's HSD test or Tamhane's T2 post hoc test (for

86 unequal variances) to reduce the risk of a Type 1 error for multiple comparisons. Results were

87 considered significant with $\mathrm{p}<0.05$.

\section{$88 \quad$ Results}

89 There were 40 participants; 17 people post-stroke undertaking exercise (SE), 13 healthy

90 exercisers (HE) and 10 participants post-stroke who were not exercising (STR). Participant

91 characteristics are shown in Table 1. As this study involved a single assessment session, no

92 participants dropped out of the study.

93 (Table 1 about here)

94 There were no significant differences in age between groups (ANOVA, $\mathrm{F}_{(2,37)}=0.791, \mathrm{p}=0.46$ )

95 although there was a non-significant trend towards more females in the HE group (9/13)

96 compared to the stroke groups $(5 / 17$ and $2 / 10)\left(\right.$ Chi-square test, Pearson $\left.X^{2}=5.632, \mathrm{p}=0.06\right)$.

97 STR participants had significantly fewer years of education (ANOVA, $\mathrm{F}_{(2,37)}=1.627$, Welch $t$

98 statistic $=3.899, \mathrm{p}=0.04)$ than both other groups and on average had suffered a stroke more

99 recently than the SE participants (Mann-Whitney Test, $U=17, \mathrm{p}<0.001$ ).

100 All participants in the HE and STR groups scored a 5 on the FAC, indicating that they were able

101 to walk independently anywhere, including stairs (with a walking aid if required). The SE group 
102 had significantly lower FAC scores (Kruskal-Wallis H (2) $=7.504, p=0.02$, Table 1 ) than both

103 other groups. There was no significant difference in the number of years exercising for the SE

104 and HE group (mean (SD) years exercising at least once per week for the SE group $=4.1 \pm 2.6$,

105 HE group $=7.2 \pm 4.5$, Mann-Whitney test, $U=3.844, p=0.14$ ).

106 The MoCA demonstrated that the mean score for all three groups was below the cut off score of

10726 indicating mild cognitive impairment. There was a significant main effect for group (ANOVA,

$108 \mathrm{~F}_{(2,37)}=4.721, \mathrm{p}=0.02$, Table 1) with the SE group score significantly lower than the HE group

$109(\mathrm{SE}$ group MoCA mean $(\mathrm{SD})=21.7 \pm 4.3$, HE group $\mathrm{MoCA}=25.8 \pm 2.1$, Tukey test, $\mathrm{p}=0.1)$;

110 there was no difference between the SE and STR groups.

111 Both stroke groups reported significantly higher values on the AQoL indicating poorer quality of

112 life (ANOVA, $\left.\mathrm{F}_{(2,37)}=3.511, \mathrm{p}=0.04\right)$ than the HE group as shown in Table 1. There was no 113 difference between the STR and SE groups.

114 After logarithmic transformation as DASS scores were positively skewed, a one way analysis of

115 variance of depression scores revealed a significant main effect for group (ANOVA, $\mathrm{F}_{(2,37)}=$

116 6.421, $\mathrm{p}=0.004$ ) and post-hoc Tukey tests confirmed that the mean (standard error) scores for

117 those in the STR group $(0.9 \pm 0.2)$ were significantly greater than both $\mathrm{SE}(0.4 \pm 0.1, \mathrm{p}=0.02)$

118 and $\mathrm{HE}(0.3 \pm 0.1, \mathrm{p}=0.004)$ groups (Figure 1). For stress there was also a significant main

119 effect for group (ANOVA, $\left.\mathrm{F}_{(2,37)}=6.367, \mathrm{p}=0.004\right)$. Participants in the STR group reported

120 significantly greater stress $(0.8 \pm 0.1)$ than both the SE $(0.4 \pm 0.1$, Tukey test, $\mathrm{p}=0.02)$ and HE

121 groups $(0.3 \pm 0.1, \mathrm{p}=0.005)$. The third subscale, anxiety, did not show a significant effect for

122 group (ANOVA, $\mathrm{F}_{(2,37)}=2.004$, Tukey test, $\mathrm{p}=0.15$ ).

Regression analysis was considered to investigate patient characteristics which may impact upon exercise and mood. There was no significant correlation between depression/stress and age, sex, 
years since stroke, functional ambulation category, years of education or cognitive status. Further, there was no correlation between the MoCA and quality of life or education. However, Pearson product-moment correlation coefficients revealed significant correlations were between DASS subscores and AQoL scores (depression $r=0.453, \mathrm{p}=0.003$, stress $r=0.387, \mathrm{p}=0.014$, anxiety $r=0.572, \mathrm{p}<0.001)$

\section{Discussion}

124 This study indicates that those exercising in a group at least once per week reported significantly

125 less depression and stress than people following stroke who were not currently exercising. While

126 we cannot claim that exercise alone is responsible for this association, it is an intriguing

127 possibility. The supervised exercise sessions were conducted in a group, so it could be that social

128 contact and support may have contributed to lowered stress and depression (Babyak et al. 2000).

129 In contrast, both stroke groups reported worse quality of life than the healthy exercisers which is

130 likely reflecting their AQoL scores for dependence on medications, regular treatment by a doctor

131 and often requiring assistance with activities of daily living.

132 There were higher scores for depression in the stroke group not taking part in exercise. Data

133 regarding use of antidepressants were self-reported, with three participants in the STR group on a

134 stable dose of antidepressants, two participants in the HE and none in the SE group. This suggests

135 that although depression was more prevalent in the STR group, more individuals in this group

136 were receiving treatment which strengthens our findings. In addition, difference in stress levels

137 between groups also followed a similar pattern. These results are based on a small sample,

138 therefore further investigation of the potential for exercise to decrease post-stroke depression and

139 stress are warranted. 
140 Health-related quality of life was lower in both stroke groups. There was no difference between

141 those who exercise and those who did not, suggesting that exercise is unlikely to mediate

142 improved mood via improved quality of life. Quality of life, as measured with the AQoL tool

143 reflects items such as dependence on others for assistance and on medications and regular

144 medical treatment. Many stroke survivors are dependent on several prescription medicines for

145 life, requiring close monitoring by their regular medical practitioner, so this is likely to have

146 influenced AQoL scores. Further we have shown that quality of life correlates with depression,

147 anxiety and stress scores, so even the stroke survivors who exercise have their quality of life

148 influenced by psychological factors.

149 There were several baseline differences between groups which may impact upon our findings.

150 Firstly, the SE group had significantly lower scores on the FAC, due to the inclusion of several

151 individuals who needed assistance with their mobility. We may expect that those with greater

152 mobility restrictions may have greater mood disturbance but this was not the case in this sample

153 of stroke survivors. The STR group also had significantly fewer years of formal education but

154 there was no evidence of lower cognitive scores in the STR group. Rather, the SE participants

155 had significantly lower scores on the MoCA, suggesting more severe cognitive impairment. This

156 is likely to be due to the duration of time since their stroke. These individuals were almost 10

157 years post-stroke and incidence of stroke is associated with an increased risk of dementia and

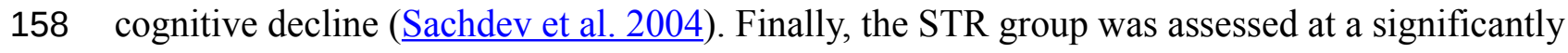

159 shorter period following their stroke, on average 1.6 years post-stroke compared to 9 years in the

160 SE group. This is unlikely to be a significant limitation as a recent multicentre trial provides

161 evidence that depression and anxiety increased significantly 5 years after stroke compared with 6

162 months post-stroke (Lincoln et al. 2012) and a systematic review of observational studies 
163 indicated that the prevalence of depression was similar in acute, sub-acute and chronic phases

164 after stroke (Hackett et al. 2005).

165 Another limitation of the study is the observational design; formal attendance was not recorded 166 and participants were free to exercise ad libitum. However, the majority of exercisers did report

167 attend regularly, 1-2 times per week, with very few absences due to illness. It is also worth noting

168 that these results are only generalizable to those individuals who chose to respond to the

169 recruitment flyer; it is possible that those people following stroke and the healthy adults with

170 lower mood states may not have chosen to take part in the study.

171 There is growing evidence to demonstrate that group exercise may influence mood following

172 stroke. A pilot study comparing group exercise alone to group exercise and yoga found similar

173 improvements in anxiety and depression between the two groups of stroke survivors (Kahn et al.

174 2008). Further, both a cardiac rehabilitation model of exercise (Lennon et al. 2008) or treadmill

175 training (Smith \& Thompson 2008) improved self-reported depression in individuals post-stroke.

176 However, another recent study suggested that combined aerobic and resistance exercise classes,

177 similar to the present study, were not effective in improving depressive symptoms (Hickey et al.

178 2012). A large randomized controlled trial recruiting stroke survivors and their families is

179 underway to address this important area of research, although exercise is just one component of

180 the planned intervention, along with cognitive behavioural therapy (Gray et al. 2011). While the

181 current study is observational in design, the strength of this study is that we have compared mood

182 states in community dwelling adults and people following stroke and can demonstrate the

183 potential benefits of exercising in a group setting to improve mood, particularly stress and

184 depression. This project provides preliminary data for proof of concept and provides justification

185 for further research using more robust clinical designs to investigate the role that exercise has on 186 mood post-stroke. 


\section{Conclusion}

188 This community based study provides some preliminary evidence that people post-stroke who 189 exercise in a group setting regularly have significantly lower stress and depression compared to 190 stroke survivors who do not exercise. Future research should focus on the precise type and 191 amount of exercise capable of improving mood following stroke, the impact of exercise in the

192 early versus late stage post-stroke and the specific contribution of group versus individual 193 exercise programs. 
195

196

197

198

199

200

201

202

203

204

205

206

207

208

209

210

211

212

213

214

215

216

217

218

219

220

221

222

223

224

225

226

227

228

229

230

231

232

233

234

235

236

237

238

239

240

Babyak M, Blumenthal JA, Herman S, Khatri P, Doraiswamy M, Moore K, Craighead WE, Baldewicz TT, and Krishnan KR. 2000. Exercise treatment for major depression: maintenance of therapeutic benefit at 10 months. Psychosom Med 62:633-638.

Byrne A, and Byrne DG. 1993. The effect of exercise on depression, anxiety and other mood states: a review. J Psychosom Res 37:565-574.

Crawford JR, and Henry JD. 2003. The Depression Anxiety Stress Scales (DASS): normative data and latent structure in a large non-clinical sample. Br J Clin Psychol 42:111-131.

Folstein MF, Maiberger R, and McHugh PR. 1977. Mood disorder as a specific complication of stroke. J Neurol Neurosurg Psychiatry 40:1018-1020.

Foundation NS. 2008. National Stroke Audit Post Acute Services. Melbourne: National Stroke Foundation.

Gray RJ, Myint PK, Elender F, Barton G, Pfeil M, Price G, Wyatt N, Ravenhill G, Thomas E, Jagger J, Hursey A, Waterfield K, and Hardy S. 2011. A Depression Recognition and Treatment package for families living with Stroke (DepReT-Stroke): study protocol for a randomised controlled trial. Trials 12:105.

Hackett ML, Yapa C, Parag V, and Anderson CS. 2005. Frequency of depression after stroke: a systematic review of observational studies. Stroke 36:1330-1340.

Haley WE, Allen JY, Grant JS, Clay OJ, Perkins M, and Roth DL. 2009. Problems and benefits reported by stroke family caregivers: results from a prospective epidemiological study. Stroke 40:2129-2133.

Hawthorne G. 2000. Using the Assessment of Quality of Life (AQoL) instrument. Victoria: Centre for Health Program Evaluation.

Hickey A, Holly D, McGee H, Conroy R, and Shelley E. 2012. Knowledge of stroke risk factors and warning signs in Ireland: development and application of the Stroke Awareness Questionnaire (SAQ). Int J Stroke 7:298-306.

Howard G, Lackland DT, Kleindorfer DO, Kissela BM, Moy CS, Judd SE, Safford MM, Cushman M, Glasser SP, and Howard VJ. 2013. Racial differences in the impact of elevated systolic blood pressure on stroke risk. JAMA Intern Med 173:46-51.

Kahn R, Robertson RM, Smith R, and Eddy D. 2008. The impact of prevention on reducing the burden of cardiovascular disease. Circulation 118:576-585.

Lees R, Fearon P, Harrison JK, Broomfield NM, and Quinn TJ. 2012. Cognitive and mood assessment in stroke research: focused review of contemporary studies. Stroke 43:16781680.

Lennon O, Carey A, Gaffney N, Stephenson J, and Blake C. 2008. A pilot randomized controlled trial to evaluate the benefit of the cardiac rehabilitation paradigm for the non-acute ischaemic stroke population. Clin Rehabil 22:125-133.

Lincoln NB, Brinkmann N, Cunningham S, Dejaeger E, De Weerdt W, Jenni W, Mahdzir A, Putman K, Schupp W, Schuback B, and De Wit L. 2012. Anxiety and depression after stroke: a 5 year follow-up. Disabil Rehabil.

Lindsay MP, Gubitz G, Bayley M, Hill MD, Davies-Schinkel C, Singh S, and Phillips SC. 2010. Canadian Best Practice Recommendations for Stroke Care (Update 2010). On behalf of the Canadian Stroke Strategy Best Practices and Standards Writing Group. 2010. Ottawa, Ontario Canada: Canadian Stroke Network.

Mehrholz J, Wagner K, Rutte K, Meissner D, and Pohl M. 2007. Predictive validity and responsiveness of the functional ambulation category in hemiparetic patients after stroke. Arch Phys Med Rehabil 88:1314-1319.

PeerJ reviewing PDF | (v2014:01:1423:1:0:NEW 5 Mar 2014) 
241 Nasreddine ZS, Phillips NA, Bedirian V, Charbonneau S, Whitehead V, Collin I, Cummings JL, 242 and Chertkow H. 2005. The Montreal Cognitive Assessment, MoCA: a brief screening tool for mild cognitive impairment. J Am Geriatr Soc 53:695-699.

National Stroke Foundation. 2010. Clinical Guidelines for Stroke Rehabilitation and Recovery. Canberra: National Stroke Foundation.

Sachdev PS, Brodaty H, Valenzuela MJ, Lorentz LM, and Koschera A. 2004. Progression of cognitive impairment in stroke patients. Neurology 63:1618-1623.

Smith PS, and Thompson M. 2008. Treadmill training post stroke: are there any secondary benefits? A pilot study. Clin Rehabil 22:997-1002.

Sturm JW, Osborne RH, Dewey HM, Donnan GA, Macdonell RA, and Thrift AG. 2002. Brief comprehensive quality of life assessment after stroke: the assessment of quality of life instrument in the north East melbourne stroke incidence study (NEMESIS). Stroke $33: 2888-2894$. 
Table 1. Participant characteristics for the three groups with mean scores and standard deviations

\begin{tabular}{|c|c|c|c|}
\hline & $\begin{array}{c}\text { Stroke and } \\
\text { Exercise (SE) }\end{array}$ & $\begin{array}{c}\text { Healthy Exercisers } \\
\text { (HE) }\end{array}$ & $\begin{array}{c}\text { Stroke no Exercise } \\
\text { (STR) }\end{array}$ \\
\hline & $\mathbf{n}=\mathbf{1 7}$ & $n=13$ & $\mathbf{n}=\mathbf{1 0}$ \\
\hline Age (years) & $70 \pm 10$ & $69 \pm 7$ & $65 \pm 9$ \\
\hline Males & 12 & 4 & 8 \\
\hline Years of education & $11 \pm 3$ & $11 \pm 4$ & $9 \pm 1^{*}$ \\
\hline Years since stroke & $8.9 \pm 6.9$ & $\mathrm{~N} / \mathrm{A}$ & $1.6 \pm 0.7^{*}$ \\
\hline FAC category & $4.4 \pm 1.1^{*}$ & $5 \pm 0$ & $5 \pm 0$ \\
\hline Exercise history (yrs) & $4.8+3.6$ & $6.8+4.3$ & $\mathrm{~N} / \mathrm{A}$ \\
\hline MoCA score & $21.7 \pm 4.3 *$ & $25.8 \pm 2.1$ & $24.4 \pm 4.1$ \\
\hline Left lesion & 8 & $\mathrm{~N} / \mathrm{A}$ & 5 \\
\hline AQoL & $28.8 \pm 7.0$ & $23.3 \pm 6.0^{*}$ & $30.0 \pm 3.1$ \\
\hline
\end{tabular}
Life. ${ }^{*} \mathrm{p}<0.05$. 
Figure 1. Mean (SEM) DASS scores for each subscale were calculated and revealed that the STR group had significantly greater depression and stress than both HE and SE groups, with no difference between scores for anxiety. 


\section{Figure 1}

DASS scores for the three groups

Fig 1. Mean (SEM) DASS scores for each subscale were calculated and revealed that the STR group had significantly greater depression and stress than both HE and SE groups, with no difference between scores for anxiety.

\section{DASS Scores}

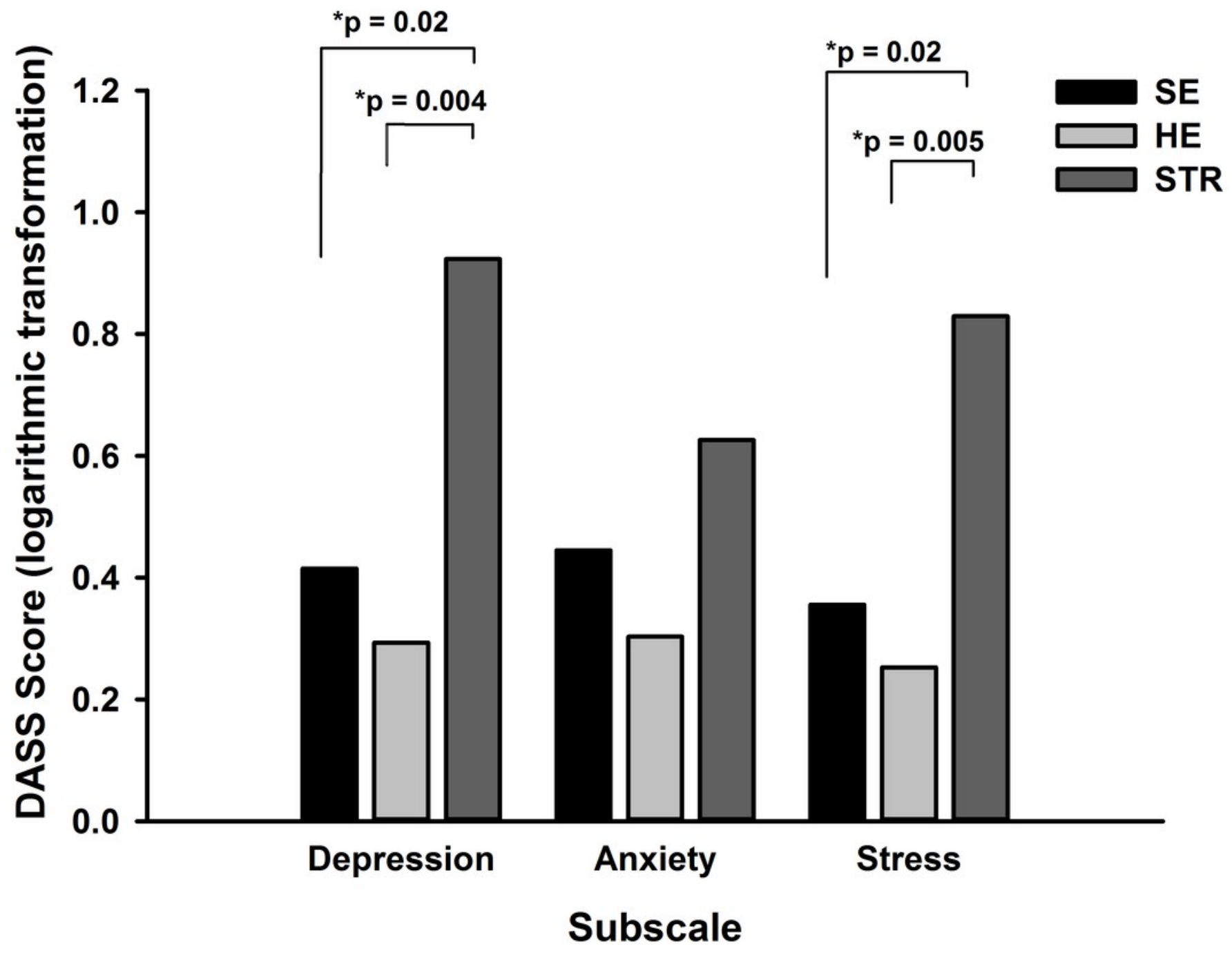

